\title{
1-Pb/s (32 SDM/46 WDM/768 Gb/s) C-band Dense SDM Transmission over 205.6-km of Single-mode Heterogeneous Multi-core Fiber using 96-Gbaud PDM-16QAM Channels
}

Kobayashi, Takayuki; Nakamura, M.; Hamaoka, F. ; Shibahara, K.; Mizuno, T.; Sano, A.; Kawakami, H.; Isoda, A.; Nagatani, Munehiko; Yamazaki, $\mathbf{H}$.

Total number of authors:

22

Published in:

Proceedings of Optical Fiber Communication Conference 2017

Link to article, DOI:

10.1364/OFC.2017.Th5B.1

Publication date:

2017

Document Version

Peer reviewed version

Link back to DTU Orbit

Citation (APA):

Kobayashi, T., Nakamura, M., Hamaoka, F., Shibahara, K., Mizuno, T., Sano, A., Kawakami, H., Isoda, A., Nagatani, M., Yamazaki, H., Miyamoto, Y., Amma, Y., Sasaki, Y., Takenaga, K., Aikawa, K., Saitoh, K., Jung, Y., Richardson, D. J., Pulverer, K., ... Morioka, T. (2017). 1-Pb/s (32 SDM/46 WDM/768 Gb/s) C-band Dense SDM Transmission over 205.6-km of Single-mode Heterogeneous Multi-core Fiber using 96-Gbaud PDM-16QAM Channels. In Proceedings of Optical Fiber Communication Conference 2017 [Th5B] Optical Society of America (OSA). https://doi.org/10.1364/OFC.2017.Th5B.1

\section{General rights}

Copyright and moral rights for the publications made accessible in the public portal are retained by the authors and/or other copyright owners and it is a condition of accessing publications that users recognise and abide by the legal requirements associated with these rights.

- Users may download and print one copy of any publication from the public portal for the purpose of private study or research.

- You may not further distribute the material or use it for any profit-making activity or commercial gain

- You may freely distribute the URL identifying the publication in the public portal 


\section{1-Pb/s (32 SDM/46 WDM/768 Gb/s) C-band Dense SDM Transmission over 205.6-km of Single-mode Heterogeneous Multi-core Fiber using 96-Gbaud PDM-16QAM Channels}

T. Kobayashi ${ }^{1}$, M. Nakamura ${ }^{1}$, F. Hamaoka ${ }^{1}$, K. Shibahara ${ }^{1}$, T. Mizuno ${ }^{1}$, A. Sano ${ }^{1}$, H. Kawakami ${ }^{1}$, A. Isoda ${ }^{1}$, M. Nagatani ${ }^{2}$, H. Yamazaki ${ }^{2}$, Y. Miyamoto ${ }^{1}$, Y. Amma ${ }^{3}$, Y. Sasaki ${ }^{3}$, K. Takenaga ${ }^{3}$, K.Aikawa ${ }^{3}$, K. Saitoh ${ }^{4}$, Y. Jung, D. J. Richardson ${ }^{5}$, K. Pulverer ${ }^{6}$, M. Bohn ${ }^{6}$, M. Nooruzzaman ${ }^{7}$, and T. Morioka ${ }^{7}$

${ }^{I}$ NTT Network Innovation Laboratories, 1-1 Hikari-no-oka, Yokosuka, Kanagawa, Japan, ${ }^{2}$ NTT Device Technology Laboratories, 3-1 Morinosato Wakamiya, Atsugi, Kanagawa, Japan, ${ }^{3}$ Fujikura Ltd., 1440, Mutsuzaki, Sakura, Chiba, Japan,

${ }^{4}$ Hokkaido University, North 14 West 9, Sapporo, Hokkaido, Japan, ${ }^{5}$ University of Southampton, Southampton, SO17 1 BJ, United Kingdom,

${ }^{6}$ Coriant R\&D GmbH, St. Martin Str. 76, 81541 Munich, Germany, ${ }^{7}$ Technical University of Denmark, DK2800 Kgs. Lyngby, Denmark Email:kobayashi.takayuki@lab.ntt.co.jp

Abstract: We demonstrate the first 1-Pb/s unidirectional inline-amplified transmission over 205.6-km of single-mode 32-core fiber within C-band only. 96-Gbaud LDPC-coded PDM16QAM channels with FEC redundancy of $12.75 \%$ realize high-aggregate spectral efficiency of $217.6 \mathrm{~b} / \mathrm{s} / \mathrm{Hz}$.

\section{Introduction}

OCIS codes: (060.1660) Coherent communications, (060.2330) Fiber optics communications

Space-division multiplexing (SDM) [1] is a promising approach to overcome the input-power limit of standard single-mode fiber and to realize over- $1 \mathrm{~Pb} / \mathrm{s}$ capacity transmission systems. Figure 1 (a) plots the transmission capacity versus transmission distance of the four SDM transmission experiments that have demonstrated over 1- $\mathrm{Pb} / \mathrm{s}$ capacity [2-5]. However all the transmission lines were unrepeated and the maximum reach was restricted to $52 \mathrm{~km}$ [2]. Furthermore, in the case over- $1 \mathrm{~Pb} / \mathrm{s}$ experiments using single-mode multi-core fibers, which is highlycompatible with conventional single-mode fiber transmission systems, both high-order modulation formats $(\geq 32$ levels) and the use of C+L-band were required [2-4]. One key to realizing cost-efficient SDM systems is to reduce the system complexity caused by the number of transceivers, which increases in proportion to the spatial multiplicity. The number of total channels versus channel speed per wavelength is shown in Figure 1 (b). The total number of channels, which corresponds to the number of transceivers, is defined as the product of SDM multiplicity and the number of WDM channels. As the channel speeds per lambda were less than $300 \mathrm{~Gb} / \mathrm{s}$ as in recent experiments [3-5], the required number of transceivers for over-1-Pb/s capacity SDM transmission systems can easily become as high as 10,000 . One solution to simplify SDM systems would be to employ higher baud rate transceivers [6, 7] to decrease the number of WDM channels in each SDM channel.

In this paper, we demonstrate the first 1- $\mathrm{Pb} / \mathrm{s}$ dense SDM (DSDM) unidirectional inline-amplified transmission experiment over 205.6-km of 32-core fiber using 100-GHz-spaced C-band WDM, utilizing a low crosstalk heterogeneous multi-core fiber [8] and by employing BCH/LDPC-concatenated-coded PDM-16QAM with 12.75\% FEC redundancy. Digital pre-processed analogue multiplexed DAC (DP-AM-DAC) [7] extends the channel speed per lambda up to $768 \mathrm{~Gb} / \mathrm{s}$. It simplifies the SDM system by reducing the number of transceivers down to 1472 with an aggregate spectral efficiency of $217.6 \mathrm{~b} / \mathrm{s} / \mathrm{Hz}$. We also show the potential of 8-dimensional 16QAM (8D16QAM) [9] for enhancing the transmission distance while keeping the base constellation size of 16QAM.
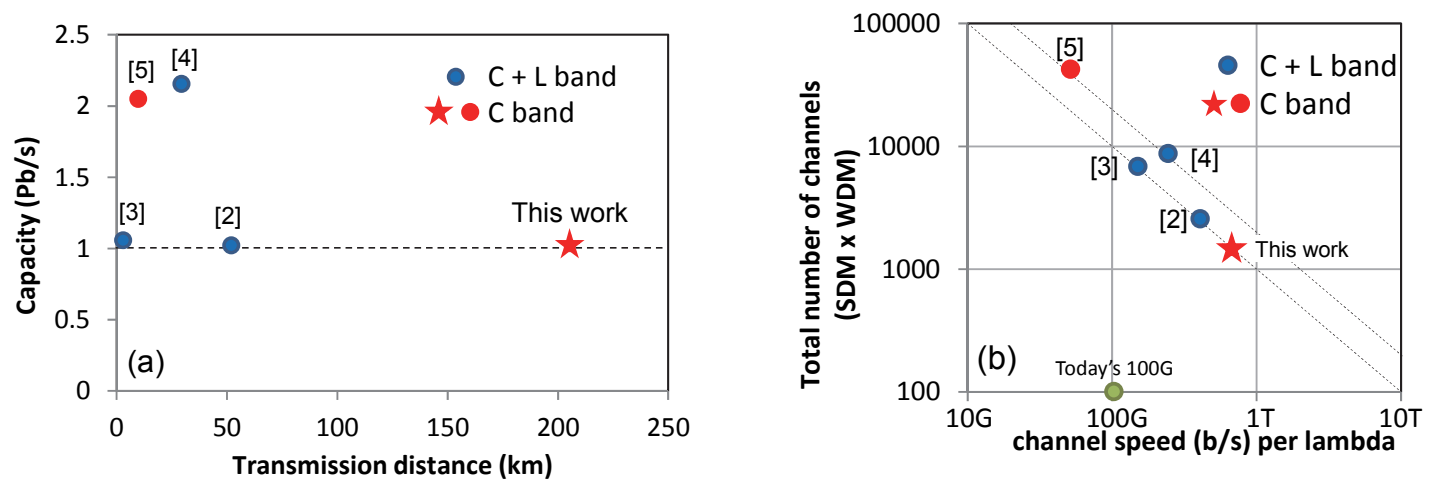

Fig.1 (a) Capacity versus transmission distance (b) Total number of channels vs channel speed/lambda from recent over- $1 \mathrm{~Pb} / \mathrm{s}$ capacity $\mathrm{SDM}$ transmission experiments 


\section{Experimental setup}

Figure 2 shows the experimental setup for the 46-channel C-band transmission. At the transmitter, $46 \mathrm{CW}$ lasers have frequencies set to the ITU-T $100-\mathrm{GHz}$ grid and these are separately multiplexed to provide even and odd channels. A tunable external cavity laser (ECL) with a linewidth of $\sim 60 \mathrm{kHz}$ was used for the test channel; the remaining 45 lasers were $\sim 2-\mathrm{MHz}$ linewidth DFB lasers. We used three IQ-modulators for test and even-/oddchannels. 96-Gbaud modulator drive signals were generated by DP-AM-DACs containing four channel sub-DACs as arbitrary waveform generator (AWG) with 32-GHz bandwidth operated at $96 \mathrm{GS} / \mathrm{s}$ and two analogue multiplexers (A-MUX) driven by a 48-GHz clock signal. The data fed into the sub-DACs was pre-processed offline. At the transmitter-side offline DSP, transmitted bits, which were derived from DC-balanced pseudo random binary sequence (PRBS) with a length of $2^{23}$, were encoded with concatenated outer BCH and inner LDPC codes that were defined according to the DVB-S2 standard [10]. The codeword length of the inner LDPC code was 64,800. The overheads of the LDPC code and $\mathrm{BCH}$ code were $12.5 \%$ and $0.22 \%$, respectively. The total FEC overhead was $12.75 \%$. Encoded bits were gray-mapped to 16-QAM symbols after training sequence (TS) insertion with $0.15 \%$ overhead. In the case of 8D-16QAM, symbol mapping followed the rule described in [9]. Pulse-shaped signals with a roll-off factor of 0.01 were pre-processed for DP-AM-DACs. After IQ-modulation, polarization multiplexing was performed by a PDM emulator with a delay line of $200 \mathrm{~ns}$. The signals were then fed into wavelength selective switches (WSSs) for optical equalization, alias removal and channel combining of even/odd channels and signal under test. This yielded 46-ch 100-GHz-spaced WDM 96-Gbaud PDM signals. A line rate of $768 \mathrm{~Gb} / \mathrm{s}$ and net data rate of $680.14 \mathrm{~Gb} / \mathrm{s}$ for LDPC-coded PDM 16-QAM were achieved. Employing 8D-16QAM, the net data rate was $510.1 \mathrm{~Gb} / \mathrm{s}$. The input signals to the 32-core fiber were derived from the delayed copies of the 46-ch WDM signals using each optical delay line with a multiple of $5 \mathrm{~ns}$ for decorrelation between the 32 cores. In the case of LDPCcoded 16QAM, the resulting 32-core aggregate capacity after subtracting the FEC and TS overheads was $1.001 \mathrm{~Pb} / \mathrm{s}$ (32SDM x 46 WDM x $680.14 \mathrm{~Gb} / \mathrm{s} /$ lambda).

The transmission line is a partial recirculating loop system as proposed in [11] for validating inline-amplified multi-core fiber transmission systems. It consists of a 51.4-km heterogeneous 32-core fiber with two types of trenchassisted core having high and low refractive index differences [8]. Its loss coefficient was $<0.24 \mathrm{~dB} / \mathrm{km}$ and its $\mathrm{A}_{\text {eff }}$ was $>80.3 \mu \mathrm{m}^{2}$. The worst crosstalk including fan-in/fan-out (FI/FO) devices when all cores were launched was $<$ $-34.5 \mathrm{~dB} / \mathrm{span}$. A loop-synchronous polarization scrambler (LSPS) was inserted into the loop of the core under test, and eight recirculating loops were allocated to the first and second nearest cores of the core under test. The span losses were compensated by C-band EDFAs only.

At the receiver $(\mathrm{Rx})$ side, an optical switch selected one of the received signals, which was wavelengthdemultiplexed by using an optical tunable filter (OTF) and detected by a polarization-diversity coherent Rx. We used a free-running ECL with a linewidth of $\sim 60 \mathrm{kHz}$ as the local oscillator. The received signal was digitized using a digital storage oscilloscope operated at $200 \mathrm{GS} / \mathrm{s}$ with $\sim 70-\mathrm{GHz}$ bandwidth. After frontend error correction, the chromatic dispersion was compensated by frequency domain equalization. Polarization de-multiplexing and signal equalization were realized by 101-tap T/2-spaced 4x2 complex adaptive FIR filters enabling precise lane-by-lane equalization and skew error correction [12]. The carrier frequency offset was compensated by a digital PLL. Adaptive DSP functions were pre-converged in data-aided mode using least-mean square (LMS) criteria and then a decision-directed LMS algorithm was employed for tracking.

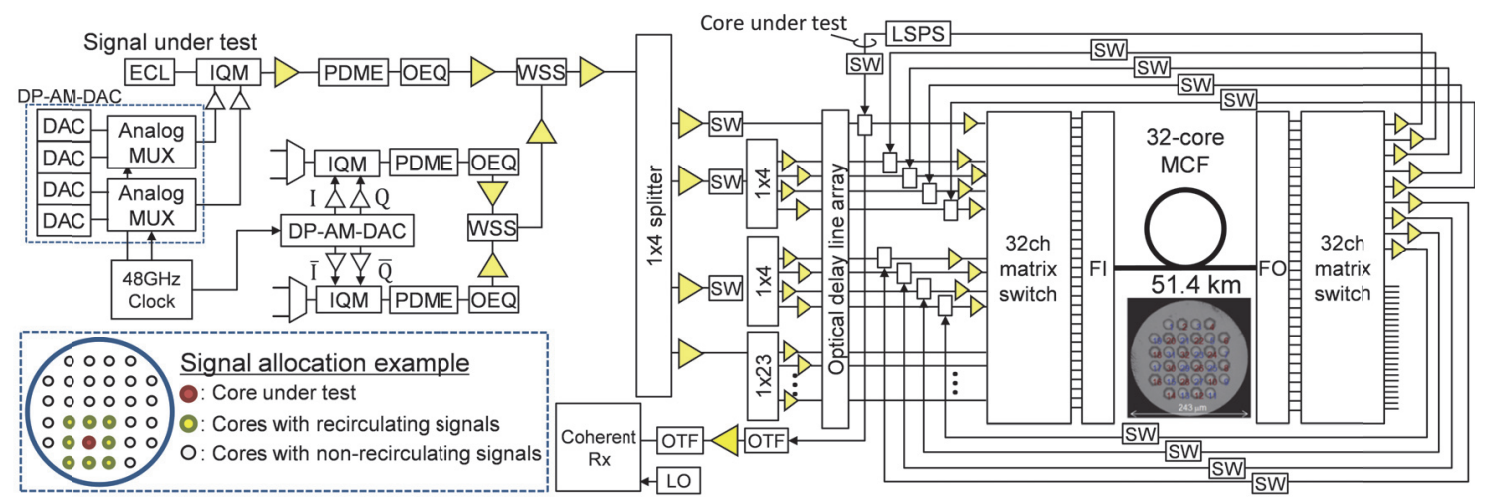

Fig.2 Experimental setup

\section{Results}

First, we investigated the error correction threshold of the concatenated BCH and LDPC codes with $12.75 \%$ overhead. Figure 3 shows the waterfall characteristic after the BCH/LDPC decoding. It was obtained from Monte 
Carlo simulations under AWGN. The FEC threshold corrected to a bit error ratio (BER) of $10^{-15}$ is estimated to be a Q-factor of $6.5 \mathrm{~dB}$, corresponding to a BER of $1.73 \times 10^{-2}$. Note that, 8D-16QAM has the same error correction threshold as PDM-16QAM because we employed the same FEC coding. Next, we measured the Q-factor dependence on transmission distance in the case of LDPC-coded PDM-16QAM and 8D-16QAM. Figure 4 shows Qfactors as a function of transmission distance at a channel of $1545.32 \mathrm{~nm}$ (ch.23) in the 46-ch C-band WDM configuration. The average fiber input power was set to $+2.9 \mathrm{dBm} / \mathrm{ch}$. The plots show averaged Q-factors of allcores and the best/worst core transmission performance. These results show that 8D-16QAM enables a flexible configuration, while maintaining the base constellation size of 16QAM, allowing a 4-fold enhancement in the transmission distance (the net data rate is reduced by 25\% compared with the LDPC-coded PDM-16QAM). Finally, we measured the 96-Gbaud LDPC-coded PDM-16QAM performance for 32-core, 46-channel SDM/WDM transmission over $205.6 \mathrm{~km}$ of MCF. The transmission distance was restricted by the EDFA's output power limitation and not by fiber nonlinearity or inter-core crosstalk. The BER was calculated from the 4 Mbit demodulated signals which were then converted into Q-factors. The received optical spectra through core\#8 span is shown in Fig.5; the inset shows the recovered PDM-16QAM constellation diagrams for X- and Y-polarization at $1545.32 \mathrm{~nm}$ for core\#8. The measured Q-factor performance after $205.6 \mathrm{~km}$ transmission is shown in Fig.6. Qfactors of all 1472 channels (46 WDM x 32 cores) were observed to be better than the FEC Q-factor threshold of 6.5 $\mathrm{dB}$ represented by a dashed line. No error was observed after the LDPC/BCH decoding for all received channels and unidirectional $1.001 \mathrm{~Pb} / \mathrm{s}$ DSDM transmission over $205.6 \mathrm{~km}$ with an aggregate spectral efficiency of $217.6 \mathrm{~b} / \mathrm{s} / \mathrm{Hz}$ was achieved.

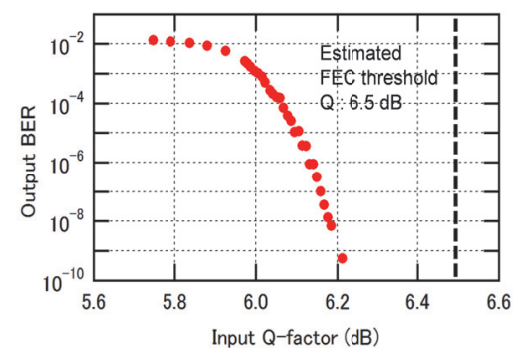

Fig.3 Water fall characteristic of FEC

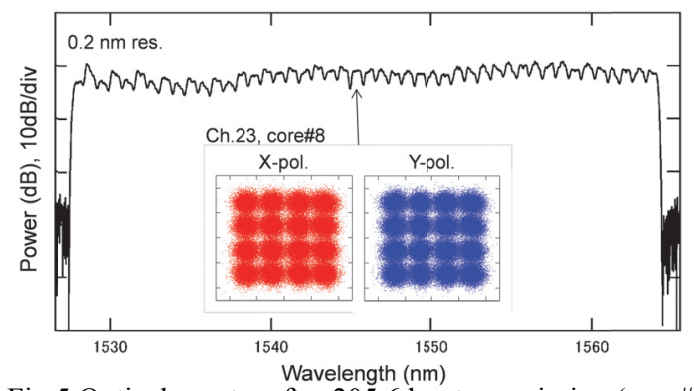

Fig.5 Optical spectra after $205.6 \mathrm{~km}$ transmission (core\#8) Insets: recovered constellations $(1545.32 \mathrm{~nm}$, core $\# 8)$

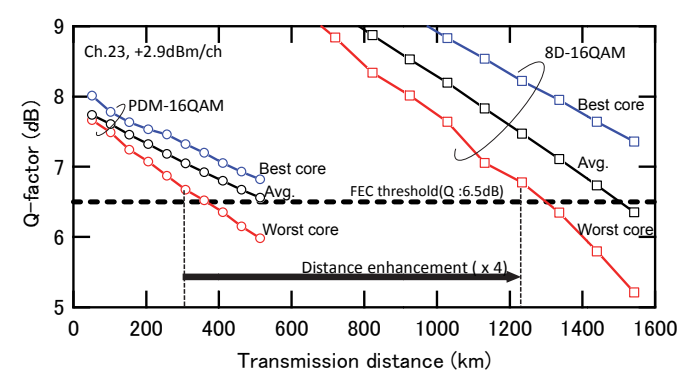

Fig.4 Q-factor dependence on transmission distance

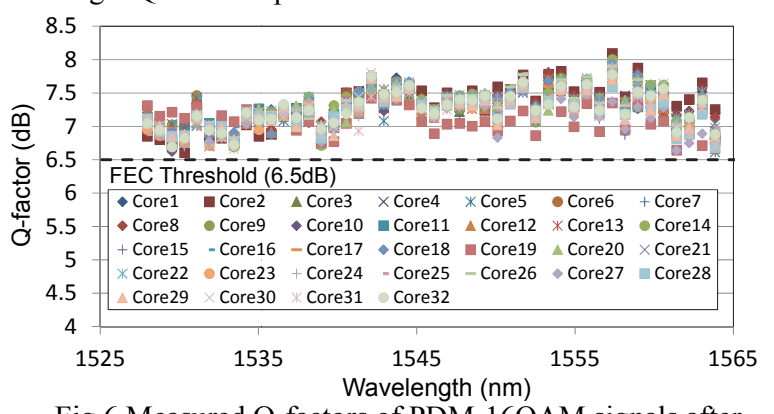

Fig.6 Measured Q-factors of PDM-16QAM signals after 205.6-km transmission

\section{Conclusion}

We have demonstrated 1-Pb/s unidirectional inline-amplified transmission over 205.6-km 32-core single-mode fiber within the C-band for the first time. A 768-Gb/s concatenated-BCH/LDPC-coded PDM-16QAM channel generated by DP-AM-DAC simplified the SDM system and its total number of channels was reduced to 1472. Employing $\mathrm{PDM}-16 \mathrm{QAM}, 1 \mathrm{~Pb} / \mathrm{s}$ capacity with an aggregate spectral efficiency of $216.7-\mathrm{b} / \mathrm{s} / \mathrm{Hz}$ was achieved. We also demonstrated the potential of the 8D-16QAM format for enhancing the transmission distance by 4 times.

Part of this research utilized results from the EU-Japan coordinated R\&D project on "Scalable And Flexible optical Architecture for Reconfigurable Infrastructure (SAFARI)" commissioned by the Ministry of Internal Affairs and Communications (MIC) of Japan and EC Horizon 2020.

\section{References}

[1] T. Morioka, proc. OECC2009, FT4 (2009).

[2] H. Takara et al., proc. ECOC2012, Th.3.C.1 (2012).

[3] D. Qian et al., proc. Frontiers in Optics 2012, FW6C.3 (2012)

[4] B. J. Puttnam et al., proc. ECOC2015, PDP3.1 (2015).

[5] D. Soma et al., proc. ECOC2015, PDP.3.2 (2015)

[6] G. Raybon et al., proc. OFC2016, Th3A.2 (2016).
[7] H. Yamazaki et al., JLT, Vol. 34, No. 7, pp.1579-1584, (2016)

[8] Y. Sasaki et al., proc. ECOC 2016, W.2.B.2,(2016)

[9] M. Nakamura et al., JLT, Vol. 35, No. 8, pp.1-7 (2017)

[10] ETSI, Tech. Report 102 376, V.1.1.1(2005)

[11] T. Mizuno et al., JLT, Vol. 35, No.3, pp.488-498, (2017).

[12] R. Rios Müller et al., JLT, Vol. 33, No.7, pp.1315-1318, (2015). 